\title{
PERCEPCIÓN DEL RESPETO Y MANTENIMIENTO DE LA DIGNIDAD EN PACIENTES HOSPITALIZADOS
}

\begin{abstract}
Beatriz Campillo', Josep Corbella², Marta Gelpi², Elisenda Martinez ${ }^{2}$, Miguel Angel Viciana², José Montiel $^{3}$, Esther Abós ${ }^{4}$, Sandra Callejón ${ }^{5}$, Milagros Alonso ${ }^{6}$

Resumen: La dignidad del paciente puede verse afectada, en el transcurso de la hospitalización, por conductas observables de los profesionales. A pesar de su importancia, existen pocos instrumentos para evaluarla. A través de un estudio transversal, descriptivo, analítico y cuantitativo, se evalúa la percepción de dignidad del paciente a partir del "Cuestionario de percepción de dignidad de paciente hospitalizado (CuPDPH)", en espańol y validado. La muestra estuvo formada por 148 hombres y 138 mujeres con una media de edad de 62.82 (DE 4.05). La mediana en las puntuaciones de los ítems (escala1-5) fue de 4,59, y la media 4,51 (DE 0.34). La media de la suma de las puntuaciones en el CuPDPH ha sido de 84.51(DE 9.3), (mín. 19-máx. 95). Los ítems de la dimensión etiquetada como "identidad" han obtenido una puntuación media de 4.85 (DE 0.04), la dimensión "consideración” 3.97 (DE 0.66). No se hallaron diferencias significativas en las puntuaciones y las variables sociodemográficas. Los resultados permiten identificar puntos fuertes y áreas de mejora en el cuidado y respeto a la dignidad de las personas hospitalizadas. Profundizar en el tema resulta una oportunidad en el camino hacia la excelencia profesional, defendiendo las competencias técnica y ética.
\end{abstract}

Palabras clave: dignidad, cuestionario, atención, integridad, respeto, vulnerabilidad

Perception of respect and maintenance of dignity in hospitalized patients

\begin{abstract}
Dignity is a fundamental concept that can be modified during hospitalization by the behavior of professionals. Despite its importance, there are few instruments to evaluate it. A transversal, descriptive, analytical and quantitative study evaluates the patient's perception of dignity based on the "Cuestionario de percepción de dignidad de paciente hospitalizado (CuPDPH)" in Spanish and validated. The sample was composed of 148 men and 138 women with an average age of 62.82 (SD 4.05). The median in the item scores (Scale 1-5) was 4.59, and the mean 4.51 SD (0.34). The mean of the sum of the CuPDPH scores was 84.51(SD 9.3), (min. 19-max. 95). The items of the dimension labeled as "identity" obtained an average score of 4.85(SD 0.04), the dimension "consideration" 3.97(SD 0.66). No significant differences were found in the scores and sociodemographic variables. The results allow us to identify highlights and areas of improvement in the care and respect for the dignity of hospitalized persons. Going deeper into the topic is an opportunity, on the way to professional excellence, defending technical competence and ethical competence.
\end{abstract}

Keywords: dignity, questionnaire, care, integrity, respect, vulnerability

\section{Percepçáo do respeito e manutençáo da dignidade em pacientes hospitalizados}

Resumo: A dignidade do paciente pode ser afetada no transcurso da hospitalização por condutas observáveis dos profissionais. Apesar de sua importância, existem poucos instrumentos para avalia-la. Mediante um estudo transversal, descritivo, analítico e quantitativo, avalia-se a percepçáo da dignidade do paciente com o "Questionário de percepção da dignidade de paciente hospitalizado (CuPDPH, sigla em espanhol)", em espanhol e validado. A amostra foi constituída por 148 homens e 138 mulheres, com uma média de idade de 62,82 (DP 4,05). Não se detectaram diferenças significativas nas pontuaçôes e nas variáveis sócio-demográficas. Os resultados permitem identificar pontos fortes e áreas de melhora no cuidado e respeito à dignidade das pessoas hospitalizadas. Aprofundar-se no tema representa uma oportunidade no caminho à excelência profissional, defendendo as competências técnica e ética.

Palavras chave: dignidade, questionário, atenção, integridade, respeito, vulnerabilidade

\footnotetext{
${ }^{1}$ Escuela Universitaria Enfermería EUI Sant Pau Universidad Autónoma de Barcelona[UAB], Comité de Ética Asistencial del Hospital de la Santa Creu I Sant Pau, Barcelona, España

Correspondencia: bcampillo@santpau.cat

${ }^{2}$ Comité de Ética Asistencial del Hospital de la Santa Creu I Sant Pau, Barcelona, España

${ }^{3}$ Medical School. Universidad Autónoma de Barcelona[UAB], Comité de Ética Asistencial del Hospital de la Santa Creu I Sant Pau, Barcelona, Espańa

${ }^{4}$ Unidad de Hospitalización de Corta Estancia Hospital de la Santa Creu i Sant Pau, Barcelona, España

${ }^{5}$ Unidad de Hospitalización de Cirugía Hospital de la Santa Creu i Sant Pau, Barcelona, España

${ }^{6}$ Departamento de Farmacología Clínica, Comité de Ética Asistencial del Hospital de la Santa Creu I Sant Pau, Barcelona, España. Departamento de Medicina, Universidad Autónoma de Barcelona [UAB], Barcelona, Espańa
} 


\section{Introducción}

El concepto de la "dignidad humana" tiene una amplia tradición filosófica. Remite a derechos fundamentales, en especial al derecho a la libertad para decidir sobre el propio cuerpo y, en definitiva, sobre la propia vida(1). En un nivel legislativo, el concepto aparece en documentos internacionales y nacionales, como la Declaración Universal de los Derechos Humanos (1948), la Ley General de Sanidad de 1986, el Convenio para la protección de los derechos humanos y la dignidad del ser humano con respecto a las aplicaciones de la Biología y la Medicina, de 1997, la Ley Básica 41/2002, reguladora de la autonomía del paciente y de derechos y obligaciones en materia de información y documentación clínica, la Carta de derechos y deberes del enfermo en relación con la salud y la atención sanitaria, de 2002, o la Declaración Universal sobre Bioética y Derechos Humanos de la UNESCO, de 2005. Además, el respeto a la dignidad de la persona viene recogido en los códigos deontológicos y éticos médicos y enfermeros, y los centros hospitalarios en su misión y visión incorporan valores de respeto, dignidad y autonomía de la persona.

A pesar de que se trata de un concepto presente en nuestro vocabulario popular, cabe preguntarse si realmente comprendemos su significado y sus implicaciones (1,2). Marley(3) señala que a la dignidad le ocurre lo que a otros conceptos a los que, a pesar de no poder proporcionar una definición universalmente aceptada, probablemente la reconocemos cuando la vemos, aunque percibimos mucho más su ausencia. Asimismo Camps(1) indica que dignidad, respeto y autonomía forman parte de un conglomerado de significados que se enriquece por la conjunción de los tres y se empobrece si lo reducimos a uno de ellos.

Su estudio empírico desde el ámbito de la salud es reciente(4) y en España muy escaso. Los primeros estudios sobre la percepción de respeto y mantenimiento de la dignidad en personas hospitalizadas aparecen alrededor de 1960(5) en pacientes psiquiátricos, incorporando posteriormente otros ámbitos de atención, como geriatría, médicoquirúrgico, cuidados paliativos, cuidados intensivos, rehabilitación o en el parto, integrando por lo tanto a varios grupos de edad y situaciones de cuidado. La mayoría de las investigaciones han sido llevados a cabo desde la disciplina de enfermería, a partir de metodologías cualitativas, con el objetivo de profundizar en el conocimiento de las experiencias y vivencias de los participantes, partiendo de observaciones directas y estructuradas o semiestructuradas a pacientes y profesionales.

En un proceso de enfermedad, la persona vive cambios físicos, emocionales y sociales, pudiendo experimentar pérdidas ante las que se adapta y modifica el concepto que tiene de sí misma. Otras personas, sin embargo, a pesar de sufrir pérdidas en sus roles y funciones, consiguen mantener su percepción de dignidad estable. Es decir, la dignidad percibida por la persona a lo largo del proceso de la enfermedad sufre modificaciones. Asimismo, se ha identificado que elementos de la personalidad, como el optimismo o la resiliencia, pueden actuar como factores protectores de este sentir(6).

Las personas al enfermar anhelamos la curación. Sin embargo, la evolución de algunas enfermedades nos posiciona ante la petición del cuidado digno, como expresión de un equilibrio en el uso de los recursos proporcionados por los avances y las tecnologías y el respeto por la integridad de la persona. El desequilibrio entre ambos genera la mayoría de los conflictos bioéticos, afectando a los principios de no maleficencia, beneficencia y autonomía. Chochinov(7) sostiene que la atención y el cuidado, el "care" y el "caring" (términos que los anglosajones recogen con tanta precisión) deberían ir de la mano. Esto es, cuanto más desarrollo tecnológico se implementa en la atención, más deberían desarrollarse competencias y habilidades que concilien la benevolencia, el respeto y la compasión.

En nuestro contexto sanitario se llevan a cabo regularmente encuestas en centros asistenciales para evaluar la satisfacción de los usuarios, recogiendo datos que identifican aspectos del trato y elementos que forman parte de la dignidad, pero no la contemplan en todas sus facetas. La literatura ha revelado la existencia de dos únicos cuestionarios $(8,9)$ en los que se evalúa la percepción de la dignidad en pacientes hospitalizados en servicios generales, pero cuyas propiedades psicométricas no están 
evaluadas y no están adaptados a la lengua espańola. En el primero de ellos, basado en el código ético enfermero italiano y la literatura, Ferri(8) identifica tres facetas: intimidad, informaciónautonomía e interacción enfermera-paciente respetuosa. El cuestionario final recoge 15 ítems en formato de pregunta, con respuestas de tipo dicotómicas. El estudio de la validez de contenido fue realizado por ocho expertos, sin evaluar ninguna otra propiedad psicométrica.

El segundo cuestionario está compuesto por 16 preguntas abiertas y cerradas que pasaron a una muestra de conveniencia de 40 pacientes. Dadas sus características, no llevan a cabo ningún análisis psicométrico. Lo interesante del cuestionario son las respuestas dadas a las preguntas abiertas y el posterior análisis de contenido efectuado con la información revertida. Los pacientes mostraron tener conceptos muy claros sobre la privacidad y la dignidad, así como la manera en que pueden verse modificados por el entorno clínico o por las conductas de los profesionales(9).

En este punto nos cuestionamos: ¿qué expresan los pacientes del entorno español y en nuestra estructura hospitalaria acerca de la dignidad? ¿Cómo puede favorecerse y cómo debilitarse la dignidad? ¿Qué hacemos o dejamos de hacer los profesionales respecto de lo que atenta o pone en riesgo la dignidad de los pacientes hospitalizados? $\mathrm{Y}$ ¡cuáles son las conductas que podemos mejorar y cuáles deberíamos abandonar. Para ello, este grupo investigador llevó a cabo un estudio cuya primera fase consistió en construir y determinar las propiedades psicométricas de un cuestionario para evaluar las percepciones sobre el respeto y mantenimiento de la dignidad en el paciente hospitalizado (CuPDPH) (en prensa). La segunda fase, objeto de este manuscrito, tiene como propósito dar respuestas e interpretar las cuestiones planteadas a la luz de las puntuaciones otorgadas por los pacientes a las conductas observadas en los profesionales que les atienden, a través de la escala construida.

\section{Objetivo}

Analizar las percepciones de los pacientes hospitalizados en relación con el respeto y mantenimiento de su dignidad, a través de las puntuaciones del
CuPDPH y sus relaciones con variables sociodemográficas.

\section{Metodología}

\section{Tipo de estudio}

Estudio transversal, descriptivo, analítico y cuantitativo de la percepción del respeto y mantenimiento de la dignidad del paciente hospitalizado, a través de las puntuaciones del CuPDPH.

\section{Ámbito y población}

Se consideró como ámbito de estudio el Hospital de la Santa Creu i Sant Pau (HSCSP) que, con un total de 619 camas de hospitalización, proporciona asistencia a cinco distritos municipales, cuya población de referencia es de 300.000 habitantes (un 25\% de la población de Barcelona).

La población diana estuvo compuesta por una muestra de conveniencia de pacientes hospitalizados en las unidades de Corta Estancia (unidad de derivación del Servicio de Urgencias) y Cirugía General.

Para el cálculo de la muestra se tuvo en cuenta la máxima indeterminación. Esto es $\mathrm{p}=\mathrm{q}=0.5 \mathrm{con}$ un intervalo de confianza del $95 \%$, para que fuera posible encontrar diferencias significativas en los análisis bivariantes.

Fueron incluidos en el estudio los pacientes mayores de 18 años, conscientes y orientados, capaces de poder leer el formulario y dar respuestas, y que aceptaron voluntariamente participar.

\section{Recogida de datos}

Se informó a los equipos asistenciales de las unidades de hospitalización seleccionadas antes de la puesta en marcha del estudio. A cada participante se le entregó una hoja informativa, en la que se explicaba el objetivo del estudio y en qué consistía su participación; el documento de consentimiento, que debía ser firmado una vez hubieran sido informados, y el formulario que contenía el cuestionario CuPDPH y variables sociodemográficas y laborales.

El formulario fue entregado por un investigador 
colaborador asignado en cada unidad el día del alta, para evitar temores de los participantes en función de las respuestas proporcionadas.

La recogida de datos se efectuó entre junio de 2018 y marzo de 2019.

\section{Variables e instrumento}

Se consideró como variable principal la percepción de respeto y mantenimiento de la dignidad del paciente hospitalizado, evaluada a través del cuestionario $\mathrm{CuPDPH}$, construido por este equipo investigador en la primera fase del estudio.

A partir de la revisión de la literatura, se propuso una definición teórica inicial sobre la que se construyeron los ítems que forman el cuestionario y que se comprobó empíricamente:

"La dignidad percibida es la suma de las percepciones que el paciente tiene en relación con los comportamientos de los profesionales que lo atienden. Integra valores de respeto e integridad a la persona, identidad, espacio e información que recibe, permitiéndole ejercer su autonomía”.

El cuestionario está formado por 19 enunciados en afirmativo y negativo en el que, con una escala Likert de cinco opciones (1-5), el participante expresa su grado de acuerdo con los comportamientos observados en los profesionales sanitarios durante su hospitalización. La consistencia interna medida a través del alfa de Cronbach resultó de 0.77. Los enunciados obtuvieron un índice de discriminación $>0.40$. La adecuación de los datos para realizar el análisis factorial se determinó a partir de la prueba de Kaiser Mayer Olkin y de la prueba de Esfericidad de Barlett, y se obtuvieron valores superiores a 0.60 y $\mathrm{p}<.000$ respectivamente. A partir del análisis de componentes principales con rotación Oblimin emergieron seis factores con valores propios superiores a 1 que explicaban el $61.03 \%$ de la varianza total. Todos ellos aportaban pesos factoriales entre 0.50-0.80. Los factores que emergieron se etiquetaron como: intimidad, integridad, identidad, información, respeto y consideración.

La puntuación total del cuestionario se obtiene mediante la suma de las puntuaciones obtenidas, previa recodificación de las puntuaciones de los ítems formulados de forma negativa (puntuación mínima 19, puntuación máxima 95).

Como variables sociodemográficas se incluyó la edad, el género, el estado civil, la situación laboral, el lugar de nacimiento, el lugar de residencia, la unidad de hospitalización, el motivo del ingreso y los ingresos previos.

\section{Análisis de los datos}

\section{Análisis univariante}

Se calculó la media y mediana de las puntuaciones totales, identificando los ítems cuyas puntuaciones se hallaban por encima y por debajo de la misma para el total del cuestionario. Se calcularon frecuencias y porcentajes de respuesta para los ítems del cuestionario y de las variables sociodemográficas.

\section{Análisis bivariante}

En el análisis bivariante se procedió al estudio de la $t$ de Student, para determinar diferencias de medias entre dos variables (cuantitativa cualitativa) y Anova para tres grupos o más. Para el análisis de las relaciones entre dos variables cuantitativas se procedió al estudio del coeficiente de correlación de Pearson.

Las diferencias entre variables fueron consideradas estadísticamente significativas con un intervalo de confianza del $95 \%$ para una $p<0.05$ en un contraste bilateral.

Para la tabulación y análisis de los datos se utilizó el Statistical Package for the Social Sciences (SPSS), versión 25.0 para Windows.

\section{Aspectos éticos}

El estudio dispone de la aprobación del comité de ética de la investigación del HSCSP, código: [IIBSP-CUP-2017-76].

Se informó verbalmente y por escrito a los participantes, y se obtuvo el consentimiento informado firmado de acuerdo con la normativa vigente. $\mathrm{Ni}$ en los formularios ni en los registros del estudio aparecieron datos identificativos de las personas. 
Acta Bioethica 2020; 26 (1): 61-72

Para proteger los formularios y los consentimientos en formato papel se entregaron en cada unidad dos carpetas, en las cuales los documentos se depositaban por separado, permitiendo su protección hasta el momento de la recogida. Los formularios se guardan en un armario cerrado con llave en el despacho del investigador principal. El tratamiento bruto de los datos solo ha sido efectuado por uno de los investigadores.

\section{Resultados}

Se entregaron 301 formularios de los cuales 12 fueron descartados por estar incompletos en más de un $10 \%$.

La muestra final estuvo formada por 148 hombres $(51,75 \%)$ y 138 mujeres $(58,25 \%)$ (tres formularios no indicaron el género), con una media de edad de 62,82 (DE 4.05) y rango de 18-98. La media de días que los participantes permanecieron ingresados fue de 8.73 (DE 10.72). Entre las causas que motivaron el ingreso en la unidad de cirugía figuraban intervenciones de neoplasias del sistema digestivo (colon y recto) y otros tumores, colecistectomía, apendicectomía y cirugía vascular. Las causas de ingreso en la unidad de corta estancia fueron por patología respiratoria y problemas derivados de la insuficiencia cardíaca. La tabla 1 recoge más datos de las variables sociodemográficas de los participantes.

Tabla 1. Características de los participantes.

\begin{tabular}{|c|c|c|c|}
\hline & & Media & $\mathrm{DE}$ \\
\hline \multirow[t]{2}{*}{ Edad } & Unidad cirugía & 59.96 & 15.31 \\
\hline & Unidad corta estancia & 65.68 & 19.35 \\
\hline \multirow[t]{3}{*}{ Días ingreso } & Unidad cirugía & 11.22 & 12.17 \\
\hline & Unidad corta estancia & 15.37 & 118.19 \\
\hline & & $\mathrm{n}$ & $\%$ \\
\hline \multirow[t]{3}{*}{ Género } & Hombre & 148 & $51.75 \%$ \\
\hline & Mujer & 138 & $48.25 \%$ \\
\hline & Total & 286 & \\
\hline \multirow[t]{5}{*}{ Estado civil } & Soltero/a & 55 & $19.10 \%$ \\
\hline & Casado/a & 139 & $48.26 \%$ \\
\hline & Separado/a & 12 & $4.17 \%$ \\
\hline & Divorciado/a & 15 & $5.21 \%$ \\
\hline & Vuido/a & 41 & $14.93 \%$ \\
\hline
\end{tabular}

\begin{tabular}{|c|c|c|c|}
\hline & En pareja & 24 & $8.33 \%$ \\
\hline \multirow[t]{6}{*}{$\begin{array}{l}\text { Situación } \\
\text { laboral }\end{array}$} & Activo /a & 82 & $28.67 \%$ \\
\hline & Jubilado/a & 150 & $52.45 \%$ \\
\hline & Baja por enfermedad & 19 & $6.64 \%$ \\
\hline & Ama de casa & 15 & $5.24 \%$ \\
\hline & Estudiante & 7 & $2.45 \%$ \\
\hline & En paro & 13 & $4.55 \%$ \\
\hline \multirow[t]{3}{*}{$\begin{array}{l}\text { Lugar de } \\
\text { nacimiento }\end{array}$} & Cataluña & 165 & 57.29 \\
\hline & Resto de España & 80 & 27.78 \\
\hline & Extranjero & 43 & 14.93 \\
\hline \multirow[t]{3}{*}{$\begin{array}{l}\text { Lugar de } \\
\text { residencia }\end{array}$} & Barcelona y provincia & 262 & 90.97 \\
\hline & Resto de Cataluña & 18 & 6.25 \\
\hline & España & 8 & 2.78 \\
\hline \multirow[t]{2}{*}{$\begin{array}{l}\text { Ingreso } \\
\text { anterior }\end{array}$} & $\mathrm{Si}$ & 226 & $79.02 \%$ \\
\hline & No & 60 & $20.98 \%$ \\
\hline \multirow[t]{12}{*}{$\begin{array}{l}\text { Causa de } \\
\text { ingreso }\end{array}$} & Neoplasia digestiva & 46 & $20 \%$ \\
\hline & Colecistectomía & 16 & $6.96 \%$ \\
\hline & Enfermedad Inflamatoria & 13 & $5.65 \%$ \\
\hline & Otros tumores & 28 & $12.17 \%$ \\
\hline & Vascular & 11 & $4.78 \%$ \\
\hline & Apendicitis & 5 & $2.17 \%$ \\
\hline & Otras cirugías & 6 & $2.61 \%$ \\
\hline & Problemas respiratorios & 48 & $20.87 \%$ \\
\hline & Gripe A & 14 & $6.09 \%$ \\
\hline & Insuficiencia cardiaca & 23 & $10.00 \%$ \\
\hline & Cardiacas & 12 & $5.22 \%$ \\
\hline & Otras infecciones & 8 & $3.48 \%$ \\
\hline
\end{tabular}

\section{Puntuaciones del CuPDPH}

La media de la suma de las puntuaciones en el CuPDPH ha sido de 84.51 (DE 9.3), con una puntuación máxima de 95 y una mínima de 49.

La dimensión que ha obtenido una mayor media en sus puntuaciones ha sido "identidad" con una media de 4.85 (DE 0.04), y la que menos la relacionada con la "consideración" 3.97 (DE 0.66).

La mayoría de los ítems han obtenido puntuaciones en todas las opciones de respuesta, siendo la opción 5 la más puntuada. La mediana se sitúa en 4,59, y la media en 4,51 (DE 0.34), con un máximo de 4.87 y un mínimo de 3.5 . 
Percepción del respeto y mantenimiento de la dignidad en pacientes hospitalizados - Beatriz Campillo et al.

Tabla 2: Puntuaciones medias por ítem y suma total ítem.

\begin{tabular}{|c|c|c|c|c|c|c|}
\hline Dimensión & & $\begin{array}{l}\text { Opción } \\
\text { Mínima }\end{array}$ & $\begin{array}{l}\text { Opción } \\
\text { Máxima }\end{array}$ & Media & $\mathrm{DE}$ & Suma \\
\hline \multirow[t]{7}{*}{ Intimidad } & $\begin{array}{l}\text { El personal me ha mirado a los ojos al } \\
\text { hablarme }\end{array}$ & 1 & 5 & 4.70 & .65 & 71.53 \\
\hline & $\begin{array}{l}\text { He dispuesto de suficiente intimidad al usar la } \\
\text { cuña o la botella }\end{array}$ & 1 & 5 & 4.57 & .82 & 65.21 \\
\hline & $\begin{array}{l}\text { El personal ha llamado a la puerta antes de } \\
\text { entrar en la habitación }\end{array}$ & 1 & 5 & 3.89 & 1.23 & 57.47 \\
\hline & $\begin{array}{l}\text { El personal ha invitado a salir a los } \\
\text { acompañantes del otro paciente antes de hacer } \\
\text { algún procedimiento }\end{array}$ & 1 & 5 & 4.50 & .91 & 68.26 \\
\hline & $\begin{array}{l}\text { El personal tomó medidas para evitar exponer } \\
\text { mi cuerpo innecesariamente }\end{array}$ & 1 & 5 & 4.61 & .80 & 69.63 \\
\hline & $\begin{array}{l}\text { He podido hablar a solas de mi situación y } \\
\text { estado de salud, tratamiento o procedimiento } \\
\text { con el personal }\end{array}$ & 1 & 5 & 4.37 & 1.01 & 65.74 \\
\hline & Total dimensión intimidad & & & 4.44 & 0.29 & 66.31 \\
\hline \multirow[t]{4}{*}{ Integridad } & $\begin{array}{l}\text { El personal ha mostrado superioridad sin } \\
\text { importarle mi opinión ni mis necesidades }\end{array}$ & 1 & 5 & 4.23 & 1.32 & 62.74 \\
\hline & $\begin{array}{l}\text { En ocasiones me he sentido tratado como un } \\
\text { objeto }\end{array}$ & 1 & 5 & 4.59 & 1.05 & 69.32 \\
\hline & $\begin{array}{l}\text { El personal que me atendió hablaba como si no } \\
\text { estuviera delante, me he sentido invisible }\end{array}$ & 1 & 5 & 4.41 & 1.18 & 67.05 \\
\hline & Total dimensión integridad & & & 4.41 & 0.18 & 66.37 \\
\hline \multirow[t]{3}{*}{ Identidad } & Me han llamado por mi nombre & 1 & 5 & 4.87 & .49 & 73.63 \\
\hline & $\begin{array}{l}\text { Siento que he sido tratado con respeto sin tener } \\
\text { en cuenta mi condición (edad, nivel cultural, o } \\
\text { país de origen...) }\end{array}$ & 1 & 5 & 4.82 & .61 & 73.26 \\
\hline & Total dimensión identidad & & & 4.85 & 0.04 & 73.45 \\
\hline \multirow[t]{3}{*}{ Información } & $\begin{array}{l}\text { He sido informado de los detalles de mi } \\
\text { procedimiento/ tratamiento/ operación }\end{array}$ & 1 & 5 & 4.69 & .63 & 71.37 \\
\hline & $\begin{array}{l}\text { El personal ha dado respuestas claras a mis } \\
\text { preguntas }\end{array}$ & 1 & 5 & 4.72 & .54 & 71.74 \\
\hline & Total dimensión información & & & 4.70 & 0.02 & 71.55 \\
\hline \multirow[t]{5}{*}{ Respeto } & $\begin{array}{l}\text { El personal ha utilizado un lenguaje respetuoso } \\
\text { sin usar apodos o formas familiares (cariño, } \\
\text { abuelo o querido) }\end{array}$ & 1 & 5 & 4.65 & .77 & 70.42 \\
\hline & $\begin{array}{l}\text { El personal ha procurado mantener mi imagen } \\
\text { corporal (me han cubierto si llevaba bata } \\
\text { abierta) }\end{array}$ & 1 & 5 & 4.49 & .94 & 68.37 \\
\hline & $\begin{array}{l}\text { He sentido que mis derechos estaban } \\
\text { protegidos con el personal que me trató }\end{array}$ & 3 & 5 & 4.82 & .44 & 73.00 \\
\hline & $\begin{array}{l}\text { El personal me ha dedicado el tiempo necesario } \\
\text { para mi atención }\end{array}$ & 2 & 5 & 4.77 & .54 & 72.58 \\
\hline & Total dimensión respeto & & & 4.68 & 0.14 & 71.09 \\
\hline \multirow[t]{5}{*}{ Consideración } & $\begin{array}{l}\text { Si en algún momento he estado preocupado } \\
\text { o he tenido miedos relacionados con mi } \\
\text { enfermedad o tratamiento, los profesionales me } \\
\text { han ofrecido la oportunidad de hablar de ello }\end{array}$ & 1 & 5 & 4.43 & .89 & 66.68 \\
\hline & $\begin{array}{l}\text { El personal me ha preguntado con quién quería } \\
\text { compartir la información sobre mi enfermedad. }\end{array}$ & 1 & 5 & 3.50 & 1.46 & 52.00 \\
\hline & Total dimensión consideración & & & 3.97 & 0.66 & 59.34 \\
\hline & Media puntuaciones todos los ítems & & & 4.51 & 0.34 & 67.89 \\
\hline & Mediana & & & 4.59 & & 69.32 \\
\hline
\end{tabular}


Las puntuaciones de 11 de los 19 ítems que constituyen el cuestionario se situaron por encima de la media.

El ítem que obtuvo mayor puntuación corresponde al enunciado: "me han llamado por mi nombre". El ítem con menor puntuación corresponde al enunciado" han llamado a la puerta antes de entrar". La tabla 2 recoge los datos relacionados con cada uno de los ítems.

Se efectuó un análisis bivariable, en puntuaciones totales, dimensiones del cuestionario e ítems para cada una de las variables sociodemográficas y laborales, no hallando diferencias significativas en las puntuaciones totales ni en dimensiones. Solo aparecen algunas diferencias significativas en algunos de los ítems entre algunos de los grupos. El análisis de la edad y los días de ingreso con las puntuaciones obtenidas en el cuestionario no identificó correlación significativa ni superior a $\mathrm{r}>.30$.

\section{Discusión}

El cuestionario CuPDPH está formado por 19 enunciados que recogen conductas observables de los profesionales. Sumar sus puntuaciones proporciona una forma de evaluar cuantitativamente la percepción del paciente acerca del respeto a su dignidad. En el análisis de su estructura interna han emergido seis dimensiones asociadas y, por lo tanto, algunos ítems que mantienen algunas correlaciones positivas moderadas y significativas entre sí. Los resultados han puesto de manifiesto una relación entre intimidad y respeto $\mathrm{r}=.438$, intimidad y consideración $\mathrm{r}=.504$; identidad e información $r=.374$, identidad y respeto $r=.417$, e información y consideración $\mathrm{r}=.330$. El análisis de las propiedades del cuestionario permite reafirmar la definición teórica propuesta en la construcción del cuestionario, que formulaba la dignidad como una suma e interrelación de diferentes conductas observables de los profesionales a lo largo de la estancia de un paciente hospitalizado. La percepción de estas conductas, en su expresión positiva, favorece el conjunto de la dignidad percibida por el paciente; mientras que su expresión negativa, un detrimento de esta.

Se ha detectado que la puntuación media de los ítems del cuestionario es de 4,51 (DE 0.34), apareciendo algunas puntuaciones de ítems y de dimensiones por debajo de este valor. De todo ello se podría interpretar que la percepción de respeto a la dignidad del paciente hospitalizado mantiene puntuaciones adecuadas a expensas de las puntuaciones de algunas de sus dimensiones. Estos resultados observados, desde una perspectiva de cuidado a personas en situación de fragilidad y de vulnerabilidad, nos posiciona ante la necesidad de analizarlos individualmente y determinar posibles estrategias de mejora.

El objetivo de este estudio ha sido identificar las conductas de los profesionales sanitarios, percibidas por las personas hospitalizadas, que permiten respetar y mantener su dignidad de los pacientes. El análisis requiere considerar conjuntamente los procedimientos asistenciales, la singularidad de la persona en un contexto de enfermedad y el entorno y la diversidad de los profesionales de la salud, ya que todos ellos se convierten en factores y agentes que pueden promover y mantener la dignidad o, por el contrario, lesionarla y vulnerarla, dando así respuesta a nuestras preguntas iniciales de investigación. Establecemos por tanto la discusión, a la luz de las dimensiones emergidas en la construcción del cuestionario y las relaciones entre los factores y agentes mencionados anteriormente.

\section{La enfermedad - elección - control - informa- ción}

Algunas de las elecciones realizadas a diario construyen nuestra vida resultan trascendentes, otras, por el contrario, solo están circunscritas al momento inmediato o las horas siguientes. La enfermedad introduce situaciones que nos instan a llevar a cabo elecciones y a tomar decisiones severas, a cuyas consecuencias nos vamos adaptando(6). Por esta razón, las personas necesitan tomar decisiones que le permitan sentir que mantienen el control de sus vidas(10); su no adopción aumenta el sentimiento de vulnerabilidad(11). Además, durante la hospitalización las elecciones relacionadas con nuestras rutinas diarias se alteran, apareciendo elementos de despersonalización(2). Los pacientes consideran que las conductas que preservan la dignidad son aquellas en las que sienten respetadas sus capacidades y autogestión, dismi- 
nuyendo el sentimiento de dependencia(11,12). Necesitan tiempo para hacerse a la idea de situaciones que pueden ser dificultosas, pero se ven empujados en las rutinas y los horarios de la uni$\operatorname{dad}(13,14)$. Su tiempo discurre entre controles, comidas, exploraciones y visitas.

Es necesario resaltar que, en este contexto, las elecciones del paciente aparecen ligadas con la información recibida de lo que le pasa, y ésta proporciona sentimientos de control y autonomía $(15,16)$. En el transcurso de la hospitalización, las personas necesitan que se les haga partícipes de lo que va a pasar durante el día o en días futuros, se les informe antes de llevar a cabo una actividad, una exploración o una prueba $(12,15,17)$. Bayés (18) explica cómo se modifica el tiempo subjetivo en las personas enfermas y cómo los profesionales hemos de ser sensibles a esta circunstancia, en relación, por ejemplo, a los tiempos de espera.

Algunos modelos representan el concepto de "dignidad" como un equilibrio entre la percepción de control y elección de conductas y las respuestas que se establecen en la relación con los otros $(19,20)$, o la interacción de valores en la percepción de sí mismo que tiene la persona(15). La exploración de algunos rasgos de personalidad, como la capacidad de resiliencia, introversión /extroversión, apertura y sociabilidad, podrían contribuir a interpretar la percepción del respeto a la dignidad(20).

De todo ello se desprende necesariamente reflexionar en torno al empoderamiento del paciente como elemento para facilitar la autonomía y el control de la persona sobre la enfermedad(21). Si lo que queremos es potenciar la autonomía, quizás debamos trabajar para facilitar elecciones y empoderar a la persona, permitiéndole que realmente se sienta dueño de sus elecciones, acompañado y asesorado siempre por los profesionales de la salud.

\section{Intimidad confidencialidad, entorno y procedi- mientos}

La media de las puntuaciones de los ítems relacionados con la intimidad se ha situado por debajo de la mediana, permitiendo identificar y reflexio- nar sobre conductas que son susceptibles de mejorar.

La hospitalización supone una modificación de nuestros espacios personales, permite a los profesionales entrar en espacios que en cualquier otro contexto sería inaceptable(2), y la forma en que usemos y entremos en estos espacios afecta la percepción de dignidad de la persona. Este nuevo espacio está constituido por una habitación compartida, en la mayoría de los casos, con un compañero desconocido las 24 horas del día y en todas las actividades de su vida diaria: la comida, la higiene, la eliminación, el descanso, las curas, las visitas profesionales y familiares, para las que únicamente dispone como elemento de aislamiento una cortina $(12,14)$. Se entra y se sale de este espacio precipitadamente, sin llamar a la puerta para indicar el acceso y, ya dentro de la habitación, se invade el espacio alrededor del paciente sentándose en su cama sin su permiso(22).

El círculo más íntimo del espacio personal lo constituye su cuerpo, al que también los profesionales tenemos acceso $(15,17)$. Los pacientes entienden que se acceda a sus cuerpos para los tratamientos necesarios; sin embargo, se sienten humillados cuando no se cierran totalmente las cortinas, no se accede con delicadeza o no se evita una exposición total $(18,22,23)$. Los resultados son consistentes con los presentados por Ferri(6), para quien la protección de la exposición del cuerpo y la intimidad en el baño obtuvieron elevadas puntuaciones, no así los ítems referidos a la privacidad a la hora de usar cuñas o botellas.

Las salas de hospitalización han gestado cambios físicos y estructurales importantes. Lejos están las salas abiertas, pero aún quedan vestigios poco creativos del modo de conducirse en algunos procedimientos, como los pases de visita médica, las entrevistas iniciales con la recogida de datos o las curas. Quizás el problema está en el uso que hacemos de los conceptos de "intimidad", "privacidad" y "confidencialidad", sin englobarlos y comprender la vinculación entre ellos. Dicho de otra manera, para que se de un acto íntimo se necesita privacidad, y todo lo que resulte de éste, verbal o no verbal, tiene carácter confidencial.

Disponemos de mecanismos extraordinarios para 
regular y proteger la confidencialidad de los datos; sin embargo, la práctica diaria pone de manifiesto grietas que abren el debate sobre la labilidad de los sistemas y del uso que hacemos de ellos. A pesar de disponer de una historia clínica informatizada, vulneramos la confidencialidad al dejar datos clínicos en notas de papel o en estaciones enfermeras, carros o pizarras - en las que se exhiben nombres de pacientes, número de cama y exploraciones pendientes-, o en pantallas de ordenadores no bloqueadas. Además, los timbres pueden ser escuchados y respondidos desde otras habitaciones y las megafonías permiten oír las respuestas proporcionadas desde las estaciones de trabajo, desde otras habitaciones o al circular por los pasillos. No solo eso, sino que también se comparte información en zonas comunes y, algunas veces, el tono de voz que se usa es eleva$\operatorname{do}(15,16,22)$.

De la misma manera, resulta incoherente la revelación de datos al compañero de habitación o a su familia si no le hacemos salir en según qué ocasiones. Nuestro "vecino" de habitación conoce nuestra condición de "diabético", "hipertenso" o "infeccioso", pero tenemos totalmente normalizado que "esto es lo que hay", dados los recursos de que disponemos. Se hace necesario cuestionarnos nuestra sensibilidad moral en el tema(12,24). El avance hacia la excelencia exige reflexionar sobre valores y virtudes profesionales(1).

El factor "intimidad" está representado por dos ítems, no solo referidos a la corporeidad sino también a aspectos emocionales. "Miran a los ojos al hablar" y "Disponer de un espacio para hablar a solas" están hablando del cuidado individual, centrado en la persona; sin embargo, la presión y las rutinas nos empujan a generar respuestas automáticas o estereotipadas. Recordemos que el estudio ha evidenciado una correlación entre las dimensiones de "intimidad" "consideración" y "respeto. Woogara y Cheraghi $(22,23)$ recogían cómo los pacientes entienden que, para poder ser respetados, es fundamental pasar tiempo con ellos.

\section{Profesionales, competencias y relaciones con el paciente}

En la dimensión de respeto, la media de puntuaciones ha resultado elevada, con puntuacio- nes medias en los ítems de 4,68 (DE 0.77), que los pacientes materializan fundamentalmente en "usar un lenguaje respetuoso", resultado similar a otros estudios sobre la dignidad $(11,12,15,25)$. El cuestionario de Ferri también demostró puntuaciones elevadas en los ítems referidos al tono en el que se dirigían las enfermeras, de forma respetuosa sin usar apodos, y a la toma de decisiones(6).

El ítem "El personal ha procurado mantener mi imagen corporal (me han cubierto si llevaba la bata abierta)" ha obtenido una baja puntuación, resultado consistente con otros estudios. Cuando un paciente ingresa, se le coloca una pulsera identificativa. El paciente pasa de tener un nombre, a disponer de un número de cama o habitación(15). En algunos centros, al paciente le colocan un pijama o camisón institucional, que merma parte de la identidad que le otorgan los adornos y los vestidos que elige $(11,22)$, y que le proporcionan bienestar y seguridad. Por otro lado, no hay que olvidar la relación de todo ello con el concepto de sí mismo y la autoestima. La visión que otorga la horizontalidad de uno, estar encamado, frente a la verticalidad de los otros, modifica la percepción que cada uno tiene de su imagen y la que el resto percibe de él. Todo ello pone una vez más de manifiesto una realidad: en el proceso de hospitalización se adquiere la "marca paciente".

Otro significado con el que se puede relacionar hace referencia a cómo los profesionales mantenemos la identidad del paciente dirigiéndonos a ellos sin respetar su individualidad, llamándoles por el nombre sin su permiso o usando términos como "querido" o "amor" (17), o no tratándoles como adultos(26), lo cual puede repercutir directamente en la información que se proporciona, tanto en forma como en contenido.

\section{Integridad, consideración y relación con los profesionales}

Los pacientes entienden que es un deber de los profesionales preservar la dignidad innata del hombre; el respeto a todos por igual, sin discriminación; el ser tratados como personas por su valor inherente y no como un objetos(16,23). Los estudios revelan que una de las experiencias más negativas relatadas por los pacientes son las vividas en las rondas de los médicos(12), cuando 
el equipo no se presenta y se sienten vistos más como un "órgano" que como personas $(11,22)$. Algunos estudios cualitativos recogen cómo los pacientes se muestran extraordinariamente sensibles a identificar, en la cara de los profesionales, expresiones no verbales de falta de consideración por los estragos causados por la enfermedad(12)o por su condición(16).

Asimismo, perciben como violaciones el sentir que no son vistos u oídos cuando tardan en dar respuesta, cuando actúan con indiferencia o muestran actitudes condescendientes o minimizantes(12).

La dimensión de la "consideración" está representada en el CuPDPH a través de dos ítems "facilitan la expresión de los sentimientos" y "me preguntan con quién quiero compartir información sobre mi enfermedad". Ambos se refieren a un profesional proactivo que permite la expresión de sentimientos. El segundo ítem, además, está identificando un elemento clave en el proceso de la información y el consentimiento: "Con quién compartir la información". Se observa que las puntuaciones en esta dimensión se sitúan por debajo de la media. En esta dimensión estamos poniendo de manifiesto en primer lugar la autonomía, la capacidad y el derecho del paciente a decidir con quién quiere compartir su proceso, a la par que estamos facilitando la expresión de sus sentimientos y preocupaciones, aspectos que sabemos posicionan al paciente en situación óptima para la toma de decisiones. Este es un derecho que los profesionales debemos tener presente y fomentar en pro de la autonomía y el fin del paternalismo como modelo de atención. Son casi veinte años de vigencia de la ley de autonomía del paciente en nuestro país y todavía se puede ver, en la práctica, situaciones en las que, por acción, por omisión o por una concepción pasiva o instrumentalizada, se pone de manifiesto que tenemos cosas que mejorar. Es necesario generar más investigación en torno al conocimiento, al uso de la ley por parte de los profesionales y a los conflictos que se presentan en la práctica $(27,28)$. Finalmente, sería deseable que los profesionales sanitarios seamos más proactivos y facilitemos al paciente la capacidad de hablar y de decidir sobre su proceso y enfermedad.

\section{Variables}

Los resultados obtenidos en este estudio ponen de manifiesto que, en la percepción de dignidad, solo aparecen algunas diferencias significativas en puntuaciones de ítems con las variables sociodemográficas examinadas, pero no en el nivel de dimensión ni para el total de la escala. Esto es, diferentes condiciones de vida, laborales, de situación familiar o de origen no son elementos que puedan discriminar diferencias significativas en el conjunto de la percepción del respeto a la dignidad. Se comparte un territorio en el que la fragilidad y la vulnerabilidad de la persona enferma y hospitalizada representan el núcleo de dicha percepción.

Puede parecer razonable pensar que el hecho de que la hospitalización se de en un contexto de urgencia o de un ingreso programado, permite al paciente cierto tiempo para poder asimilar y adaptarse a todo lo que le acontece. Sin embargo, en nuestros resultados vemos que no aparecen diferencias significativas en la percepción de dignidad con relación al motivo de ingreso. De igual modo, tampoco los reingresos han permitido identificar diferencias significativas para el total del cuestionario ni para cada una de las dimensiones. Lo cual permite proponer la universalidad de la fragilidad de la persona en torno a la condición de estar enferma. Los seres humanos compartimos territorios comunes de fragilidad y vulnerabilidad.

Esto nos posiciona ante un nuevo hallazgo: hemos puesto de manifiesto empíricamente un factor que puede ser favorable a la hora de generar intervenciones de mejora al representar a un concepto tan universal. Cualquier propuesta de mejora que se lleve a cabo va a beneficiar al conjunto de la dignidad percibida, sea cual sea la condición del paciente.

Como última consideración, retomamos palabras de Bayés(29), quien señala que cuando el deterioro que provoca la enfermedad y la percepción de recursos es inferior a la de las capacidades, el sufrimiento se instala. En ocasiones, al disminuir las funciones corporales y las actividades diarias, aumenta la sensación de pérdida de control y de integridad, del valor de la propia vida, de la autoestima de su personalidad y de su identidad(10), 
y de que la vida sin dignidad ya no es digna de vivir. Razón por la cual las conductas de los profesionales resultan fundamentales para el restablecimiento de ese equilibrio. Los pacientes reconocen fácilmente a los profesionales con una enorme capacidad para intuir lo que necesitan, a veces sin preguntar, y agradecen sobre todo la presencia constante(12) y la repuesta rápida a sus necesidades(15). Son capaces de identificar a aquellos profesionales que, en su cuidado, proporcionan "un extra", algo que no todos los profesionales ofrecen, y que incrementa el sentimiento de recibir un buen cuidado, un cuidado digno(12).

\section{Limitaciones}

No haber identificado por separado grupos de profesionales, puede haber constituido un elemento de confusión. Nuestro objetivo, sin embargo, no estaba en identificar quién sino qué estamos haciendo para poder. Se considera también la homogeneidad de la muestra, al estar considerado un único centro.

\section{Agradecimientos}

Los autores agradecen a todas las entidades y personas del Hospital de la Santa Creu i Sant Pau que han hecho posible este proyecto. También desean agradecer al personal de enfermería por su participación en la recolección de datos, a Ignasi Gigh por su colaboración y a todos los miembros del Comité de Ética Clínica por su participación como expertos. Un sincero agradecimiento a todos los pacientes que amablemente nos proporcionaron información; les deseamos lo mejor.

Conflicto de intereses: Los autores declaran que no tienen conflictos de interés.

Financiación: Este proyecto ha sido financiado con una subvención de la Fundación Grífols. La entidad financiadora no ha participado en el diseño del estudio ni en la recogida, análisis e interpretación de los datos, ni en la redacción del manuscrito.

\section{Referencias}

1. Camps V. Dignidad y autonomía. En: Boladeras M, editor. ¿Qué dignidad? Filosofía, Derecho y práctica sanitaria. Barcelona: Proteus; 2010.

2. Ridgway V. Dignity. In: Whitehead E, McIntosh A, Bryan A, Mason T, editors. Key concepts in nursing. London: Sage; 2008. p. 103-14.

3. Marley J. A concept of dignity. In: Cutcliffe J, McKenna H, editors. The essential concepts of nursing. Elsevier Ltd; 2005. p. 77-91.

4. Zahran Z, Tauber M, Watson H, Coghlan P, White S, Procter S, et al. Sistematic review: what interventions improve dignity for older patients in hospital? J Clin Nurs [Internet]. 2016; 25: 311-21. Available from: https://doi. org/10.1111/jocn.13052

5. Whitehead J, Wheeler H. Patients' experiences of privacy and dignity. Part 1: a literature review. Br J Nurs [Internet]. 2008; 17(6): 381-5. Available from: https://doi.org/10.12968/bjon.2008.17.6.28904

6. Van Gennip IE, Pasman HRW, Oosterveld-Vlug MG, Willems DL, Onwuteaka-Philipsen BD. Dynamics in the sense of dignity over the course of illness: A longitudinal study into the perspectives of seriously ill patients. Int J Nurs Stud [Internet]. 2015; 52(11): 1694-704. Available from: https://doi.org/10.1016/j.ijnurstu.2015.06.010

7. Chochinov HM. Dignity in care: Time to take action. J Pain Symptom Manage [Internet]. 2013; 46(5): 756-9. Available from: http://dx.doi.org/10.1016/j.jpainsymman.2013.08.004

8. Ferri P, Muzzalupo J, Di Lorenzo R. Patients' perception of dignity in an Italian general Hospital: A cross sectional analysis. BMC Health Serv Res [Internet]. 2015; 15(41). Available from: https://doi.org/10.1186/s12913-015-0704-8

9. Whitehead J, Wheeler H. Patients' experience of privacy and dignity. Part 2: An empirical study. Br J Nurs [Internet]. 2008; 17(7): 458-64. Available from: https://doi.org/10.12968/bjon.2008.17.7.29067

10. Rodríguez-Prat A, Monforte-Royo C, Porta-Sales J, Escribano X, Balaguer A. Patient Perspectives of Dignity, Autonomy and Control at the End of Life: Systematic Review and Meta-Ethnography. PLoS ONE 1 [Internet]. 2016; 11(3): e0151435. Available from: https://doi.org/10.1371/journal.pone.0151435

11. Narayan K, Hooker C, Jarrett C, Bennett D. Exploring young people's dignity: A qualitative approach. J Paediatr Child Health [Internet]. 2013; 49(11): 891-4. Available from: https://doi.org/10.1111/jpc.12430

12. Moen EK, Nåden D. Intensive care patients' perceptions of how their dignity is maintained: A phenomenological study. 
Percepción del respeto y mantenimiento de la dignidad en pacientes hospitalizados - Beatriz Campillo et al.

Intensive Crit Care Nurs [Internet]. 2015; 31(5): 285-93. Available from: http://www.sciencedirect.com/science/article/ pii/S0964339715000221

13. Manookian A, Cheraghi MA, Nasrabadi AN. Factors influencing patients' dignity: A qualitative study. Nurs Ethics [Internet]. 2014; 21(3): 323-34. Available from: http://nej.sagepub.com/cgi/doi/10.1177/0969733013498526

14. Walsh K, Kowanko I. Nurses 'and patients' perceptions of dignity. Int J Nurs Pract [Internet]. 2002; 2-6. Available from: https://doi.org/10.1046/j.1440-172X.2002.00355.x

15. Lin YP, Tsai YF, Chen HF. Dignity in care in the hospital setting from patients' perspectives in Taiwan: A descriptive qualitative study. J Clin Nurs [Internet]. 2011; 20(5-6): 794-801. Available from: https://doi.org/10.1111/j.13652702.2010.03499.x

16. Lin YP, Tsai YF. Maintaining patients' dignity during clinical care: a qualitative interview study. J Adv Nurs [Internet]. 2010; 67(2): 340-8. Available from: https://doi.org/10.1111/j.1365-2648.2010.05498.x

17. Henderson A, Van Epps M, Pearson K, James C, Henderson P, Osborne Y. Maintenance of patients' dignity during hospitalization: Comparison of staff-patient observations and patien feedback through interviews. Int J Nurs Pract [Internet]. 2009; 15: 227-30. Available from: https://doi.org/10.1111/j.1440-172X.2009.01764.x

18. Bayés R. Tiempo y enfermedad. Estud Psicol [Internet]. 2002; 23(1): 101-10. Available from: https://doi. org/10.1174/021093902753535213

19. Matiti M, Trorey GM. Patients'expectations of the maintenance of their dignity. J Clin Nurs [Internet]. 2008; 17: 270919. Available from: https://doi.org/10.1111/j.1365-2702.2008.02365.x

20. Matiti M, Trorey G. Perceptual adjustment levels: patients' perception of their dignity in the hospital setting. Int J Nurs Stud [Internet]. 2004; 41: 735-44. Available from: https://doi.org/10.1016/j.ijnurstu.2004.02.008

21. Boman LE, Sandelin K, Wengström Y SC. Patients' participation during treatment and care of breast cancer - a possibility and an imperative. Eur J Oncol Nurs. 2018; Dec(37): 35-42.

22. Woogara J. Patients' right to privacy and dignity in the NHS. Nurs Stand [Internet]. 2004; 19(18): 33-7. Available from: https://journals.rcni.com/doi/abs/10.7748/ns2005.01.19.18.33.c3783

23. Cheraghi M, Manookian A, Nasrabadi A. Patients'lived experiences regarding maintaining dignity. J Med Ethics Hist Med [Internet]. 2015; 8(6). Available from: https://www.ncbi.nlm.nih.gov/pmc/articles/PMC4920979/

24. Ebrahimi H, Torabizadeh C, Mohammadi E, Valizadeh S. Patients' perception of dignity in Iranian healtcare settings: a qualitative content analysis. J Med Ethics [Internet]. 2012; 38: 723-8. Available from: http://dx.doi.org/10.1136/ medethics-2011-100396

25. Pulkkinen M, Junttila K LL. The perioperative dialogue - a model of caring for the patient undergoing a hip or a knee replacement surgery under spinal anaesthesia. Scand J Caring Sci [Internet]. 2016; 30(1): 145-53. Available from: https://doi.org/10.1111/scs.12233

26. Heijkenskjold KB, Ekstedt M, Lindwall L. The patient's dignity from the nurse's perspective. Nurs Ethics [Internet]. 2010; 17(3): 313-24. Available from: http://nej.sagepub.com/cgi/doi/10.1177/0969733010361444

27. Arenas Lopez A, Castellano Arroyo M, Miranda León MT, Reche Molina A. Conocimiento y cumplimiento de los profesionales sanitarios del derecho del paciente a la información clínica. Rev Española Med Leg. 2012; 38(1): 11-6.

28. Fernández Garrido C, Lopera Uribe GE, Méndez Pérez L, Otero Espiga A, Gallardo Abril MG, Lagoa Elías C. Conocimiento de la ley de autonomía del paciente por el personal médico y de enfermería implicado en el proceso quirúrgico. Enfermería Clínica. 2009; 19(6): 330-4.

29. Bayés R. Psicología del sufrimiento y de la muerte [Internet]. Vol. 29, Anuario de psicología. Paidós; 1998 [cited 2017 May 23]. 5-17 p. Available from: http://www.raco.cat/index.php/AnuarioPsicologia/article/view/61497/88344

Recibido: 7 de noviembre de 2019

Aceptado: 10 de febrero de 2020 